\title{
Open-Minded, Not Naïve: Three-Month-Old Infants Encode Objects as the Goals of Other People's Reaches
}

\author{
Brandon Woo,2 (bmwoo@g.harvard.edu), Shari Liu ${ }^{2,3}$ (shariliu@mit.edu), \\ and Elizabeth Spelke, ${ }^{1,2}$ (spelke@wjh.harvard.edu) \\ ${ }^{1}$ Department of Psychology, Harvard University, Cambridge, MA 02138 \\ ${ }^{2}$ Center for Brains, Minds, and Machines, Cambridge, MA 02139 \\ ${ }^{3}$ Department of Brain and Cognitive Sciences, Massachusetts Institute of Technology, Cambridge MA 02139
}

\begin{abstract}
When people act on objects, their goals can depend on the objects' intrinsic properties and conventional uses (e.g., using forks, not knives, to eat spaghetti), locations (e.g., clearing the table, regardless of what is on it), or both (eating with the fork next to your plate, not your dining partner's). For adults, objects' intrinsic properties matter more than their locations in most action contexts. Whereas 5-month-old infants privilege objects' intrinsic properties in attributing goals to people reaching for objects, 3-month-old infants do not. Do younger infants fail to view reaching as goal-directed, or are they uncertain which properties of objects are relevant in different contexts? Here we show that 3-month-old infants attribute goals to others' reaching actions when given information that their actions depend on what, not where, an object is. Our findings suggest that 3-month-old infants can learn about others' object goals, before they reach for objects themselves.
\end{abstract}

Keywords: infancy; action understanding; cognitive development

\section{Introduction}

The ability to represent others' actions as goal-directed is an important prerequisite for learning from others, cooperating with others, predicting others' future actions, and inferring others' beliefs, preferences, and intentions (Baker et al., 2009; Jara-Ettinger et al., 2020; Tomasello et al., 2005). As adults, we readily infer people's goals from their actions. If a person reaches for a teddy bear over a ball, for instance, we infer that the person likely intended to contact the bear itself, rather than whatever object stood at the bear's location. Here we probe the developmental origins of the ability to represent others' actions as directed to objects and as dependent, in most cases, on objects' intrinsic properties and affordances (i.e., what objects are) rather than their locations (i.e., where objects are).

\section{Infants' Understanding of Object-Directed Reaches}

In a classic study, Woodward (1998) found that 5-, 6- and 9-month-old infants appreciate that others are likely to have object-directed goals that privilege properties that indicate what an object is rather than where it is. Specifically, infants who are habituated to a person reaching for and grasping a teddy bear over a ball expect that, if the bear and the ball switch locations, the person will continue reaching for the bear (the original object that she had reached for before, in a different location) over the ball (a different object in the location that the person reached to before). This finding, replicated many times in many labs (e.g., Biro \& Leslie, 2007; Daum et al., 2012; Feiman et al., 2015; Hernik \& Southgate, 2012; Luo \& Johnson, 2009), has inspired over two decades of research on the nature and development of these expectations.

\section{Why Do Prereaching Infants Struggle to Infer the Goal of Other People's Reaching Actions?}

The expectation that others will reach for objects based on objects' intrinsic, location-invariant properties has been found to develop between 3 and 5 months of age. When tested in the same paradigm of Woodward (1998), 3-month-old infants, who cannot reach for and pick up objects without assistance, look equally following a reach to a different object and to the same object: They do not expect that someone who has reached for a teddy bear over a ball will continue doing so after the objects switch locations (Gerson \& Woodward, 2014a, 2014b; Sommerville et al., 2005). On one view, infants come to appreciate that others have object-directed goals based on their own motor experience reaching for and grasping objects (e.g., Gallese \& Goldman, 1998; von Hofsten, 2004; Woodward, 2009). Consistent with this view, most infants begin to reach for objects between 4 and 5 months of age (Woodward, 1998). This view, however, leaves open what prereaching infants have yet to learn about other people's actions.

One possibility is that prereaching infants may know that people direct their actions to goal objects, but they may not yet know that reaching and contacting an object - the only action in the original Woodward paradigm - is a prelude to causal, intentional actions on the object. Evidence in support of this possibility comes from studies of how prereaching infants reason about the efficiency of reaching actions. Threemonth-old infants expect agents to act efficiently when their actions cause a change in the state of the object they reach for, but not when that object undergoes no change in state on contact with the hand (Liu et al., 2019; Skerry et al., 2013). Thus, prereaching infants are capable of representing the object-directed goals of others' reaching actions when those actions have observable effects on objects.

Even if reaching is seen as causal, the goal of a person reaching for a teddy bear over a ball may still be ambiguous: In the studies of Woodward (1998), Sommerville et al. (2005), and Gerson and Woodward (2014a, 2014b), infants 
were habituated to displays involving a person who reached not only for the same object, but for the same object in the same location. Was the person reaching for an object because of what the object was, where it was, or both? If young infants are uncertain about which of these construals was more important to the goal of the person who reaches for an object, then they may find both reaches to a new object and reaches to a new location to be equally novel when the objects switch places for the test phase of these studies.

Both these accounts - that infants do not see reaching as a causal intentional action, or that they are open-minded about the goals of reaching - predict that 3-month-old infants' goal attributions will improve if the events of the Woodward paradigm are altered to provide more information. The first account predicts that providing infants with more evidence that reaching is an intentional causal action will help them infer that the person's reach is object-directed. The second account predicts that disambiguating an object's intrinsic properties from its location in habituation will allow prereaching infants to infer the person's goal in reaching for the object.

To test these accounts against the prevailing account that prereaching infants do not see reaching as goal-directed at all when tested in the Woodward paradigm, we conducted two experiments with 3-month-old infants, in which (i) the object changed its state on contact with a person's hand, providing evidence that the action was causally efficacious (both experiments); and (ii) the object's intrinsic properties and location were disambiguated, by varying the location of the goal object (Experiment 2).

\section{Experiment 1}

In Experiment 1, we tested whether 3-month-old infants attribute object-directed goals to a person if the person causes objects to light up and play a sound on contact (as in Liu et al., 2019). Infants were habituated to displays depicting a person who repeatedly reached for and contacted one of two objects, simultaneous with a change in the objects' color and the onset of a sound. In test trials, the objects switched locations, and infants' looking time was recorded as the person alternately reached for each object.

Although both objects changed colors upon being touched, and the accompanying sound could have come from either object, past work provides evidence that 3-month-old infants only see a person as having caused a change in an object if physical contact occurs (Liu et al., 2019). If 3-month-old infants struggle in the Woodward paradigm because they do not understand that reaching and touching an unresponsive object is a prelude to causal, intentional action, but they privilege what an object is over where the object is in inferring the goal of a reach, then this manipulation should lead them to perform like older infants: They should look longer in test trials when the person reaches for a different object rather than the original object. In contrast, if 3-monthold infants are equally open to the possibilities that goals are defined over an object's intrinsic properties or location, or do not see reaching as goal-directed at all, then infants should look equally to the reach for the different object and to the reach for the original object, as in previous experiments.

\section{Method}

Hypotheses, methods, and analysis plans were preregistered on the Open Science Framework. All preregistration documents and stimuli can be found at https://osf.io/g7p3k/.

Participants Twenty 3-month-old infants contributed data (12 girls, 8 boys; mean age $=3.47$ months; range $=3 ; 2$ to 3;29). Three more participants began the experiment but were not included in the final sample due to fussiness that ended the experiment $(n=1)$ or experimenter error $(n=2)$. All experimenters and observers were unaware of the particular events infants saw (e.g. which object the person reached for, which test trial came first), and thus made decisions to exclude individual trials or participants without knowledge of the data they contributed.

Our sample size was based on a power analysis over pilot data (see preregistration for details).

Displays All events depicted a person reaching for one of two objects (see Fig. 1A). There were 6 to 12 habituation trials, 1 pretest trial, and 6 test trials. All habituation trials began with a person seated in front of two objects: a brown teddy bear on one side, and a red ball on the other side. The person looked to one of the objects, reached for it, and touched it with her hand. Upon contacting the object, both objects became blue and a bell sound played for 2.1 seconds, as though the person's actions had caused these changes in the object that she touched. Then, the person retracted her hand, and the objects returned to their normal colors and the sound stopped.

After the last habituation trial, a familiarization trial presented the teddy bear and the red ball, with their locations switched relative to habituation, for 15 seconds. Test trials followed, with the person seated in front of the two objects, each now in its new location. In alternating trials, the person reached for either the original object, now in a different location ("different location" trials), or a different object, located where the person had reached during habituation ("different object" trials).

Procedure Data collection was completed before the pandemic and took place in a lab environment. Infants sat in a car seat before a 60 by 40 -inch projector screen. Caregivers were seated behind their infants and asked to not speak or influence their infants' behavior.

Infants saw the same habituation video (with the person reaching to the same object, in the same location) repeatedly until they met the habituation criterion: either completion of 12 trials, or completion of three consecutive trials in which looking was no more than half as long as on the first three trials. Then infants saw the pre-test trial, which lasted for a maximum of $20 \mathrm{~s}$, and infants were required to look at it for at least $5 \mathrm{~s}$ to move on to the test phase. Lastly, infants saw 3 pairs of test trials, presented in alternating order. 

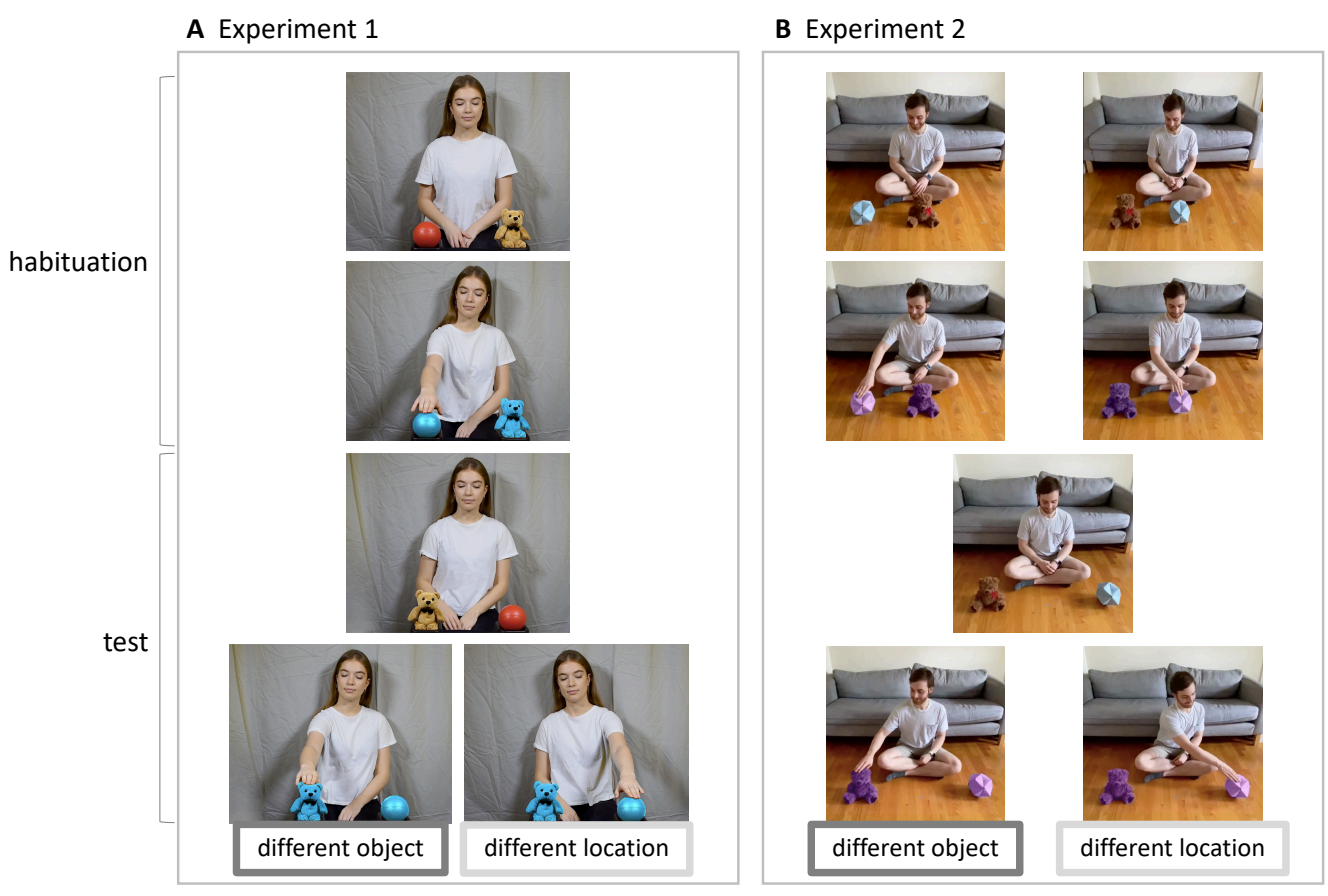

Figure 1. Stimuli in Experiments 1 and 2. In Experiment 1's habituation trials (A), a person repeatedly reached for one of two objects, which were always in the same locations. Then, at test, the two objects switched locations, and the person either reached for a different object (in a familiar location), or for the original object that she had reached for during habituation (in a different location). In Experiment 2's habituation trials (B), a person repeatedly reached for one of two objects, which switched locations between habituation trials. Then, at test, the person either reached for a different object (in a location that he had sometimes reached to in habituation), or for the old object that he had reached for during habituation (in a different location).

A coder who was unaware of which object the person reached for, and the order of the test events, coded infants' looking to the screen using the coding program PyHab (Kominsky, 2019), from the time that the videos started until the end of the trial, when infants had looked away for 2 consecutive seconds, or 45 total seconds had elapsed. A second coder recoded $100 \%$ of test trials offline using Datavyu (2014) software using the same thresholds. The intraclass correlation coefficient between the two observers' data was $0.88(95 \%$ CI $[0.83,0.91])$.

Counterbalancing The following were counterbalanced across infants: the identity of the original object that the person reached for (ball or bear), the starting location of the original object (left or right), and the order of test trials (first reach to the original object vs. first reach to the new object).

\section{Results}

Preregistered Analysis All reported $p$-values are two-tailed. To determine whether infants looked longer in test trials following a reach for a different object or to a different location, we examined looking times in a mixed-effects model. The dependent variable was log-transformed looking time. The fixed effect was trial type (different location vs. different object). The random intercepts were trial type and trial number, with a by-participant random slope for trial type.

We found that infants looked equally on the test trials presenting reaches for the different object (mean diff object $=$ 21.53 seconds) and reaches for the original object in a different location (mean diff location $=21.30$ seconds) trials $(\beta=$ $-0.03,95 \%$ of $\beta[-0.35,0.28], b=-0.02, t=-0.20, p=.843)$.

\section{Discussion}

In Experiment 1, we asked whether adding evidence that reaching is a causal intentional action (as in Liu et al., 2019) in the Woodward paradigm is sufficient to help infants infer that other people reach for objects based on what, not where, objects are. We found that this change was not sufficient for success in this task, at least at the group level: As in past research, the distribution of infants' looking preferences during the test trials centered on equal looking after the person reached for a different object and to a different location at test. This finding suggests that infants' previous failures are not simply due to a failure to understand that people intend to cause a change in objects by reaching for and grasping them.

In Experiment 2, we test whether this null result, and the other results it replicates, are explained by infants' failure to appreciate that reaching is goal-directed, or by their failure to expect that an object's intrinsic properties are more likely to 


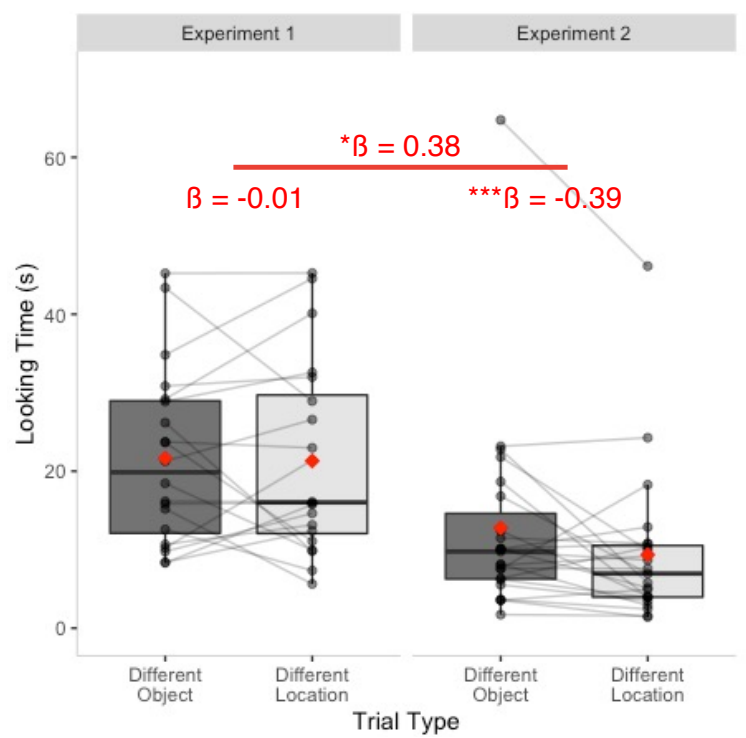

Figure 2. Results in Experiments 1 and 2, depicting the amount of time infants looked to different location and different object test trials. Red diamonds indicate means. Pairs of connected dots indicate data from a single infant. Horizontal lines within boxes indicate medians, and boxes indicate interquartile ranges. The beta coefficients (B) indicate standardized effect sizes. Asterisks indicate significant differences, following corrections for multiple comparisons $\left({ }^{*} p<.05,{ }^{* * *} p<.001 ; 2\right.$-tailed $)$.

be relevant to people's goals than is the object's location. Experiment 1, and past studies, presented infants with confounded evidence for the person's goal: 3-month-old infants may look equally to a change in the location of the reach and a change in the object of the reach, because they are uncertain whether the actor reached for the object because of its intrinsic properties or its location. By 5 months, however, infants may come to appreciate that reaches are more likely to be directed at an object based on what it is (this teddy bear) than where it is (whatever object is here). In Experiment 2, therefore, we ask whether 3-month-old infants will infer that an actor reached for and acted on a particular object, irrespective of its location, when presented with evidence that dissociates the goal object's intrinsic properties from its location.

\section{Experiment 2}

In Experiment 2, infants were again tested in the Woodward paradigm, modified as in Liu et al. (2019) to convey causal information about the reach (i.e., the actor's hand changed an object's state on contact). We asked whether infants can learn that a person is reaching to a specific object, not to an object at a specific location, when a person reaches for the same object across a change in its location. If 3-month-old infants, prior to first-person experience reaching for objects, understand that people have goals but are open-minded about whether goals are more likely to be defined over an object's intrinsic properties or its location, then the actions they viewed during habituation might inform them that the person's goal was to obtain a particular object. Thus, in test trials, infants should expect the person to reach for the original object in the new location, and they should look longer when the person reaches for the other object. In contrast, if 3-month-old infants struggle to represent objectdirected goals, even given clear evidence that a person sought a particular object regardless of its location, then they should look equally at the two test events, as they did in Experiment 1 and in past studies in which 3-month-old infants failed to attribute object goals to a person who reached to a specific object and location.

\section{Method}

Hypotheses, methods, and analysis plans were preregistered on the Open Science Framework. All preregistration documents and stimuli can be found at https://osf.io/g7p3k/.

Participants Twenty-four 3-month-old infants contributed data (9 girls; mean age $=3.42$ months; range $=3 ; 00$ to $4 ; 00$ ). An additional 2 participants began the experiment but were not included in the final sample due to fussiness.

Our sample size was based on a power analysis over pilot data (see preregistration for details).

Displays We modified the displays from Experiment 1 to provide infants with clear evidence that someone was reaching for a specific object based on what, not where, the object was (see Fig. 1B). To accomplish this in the context of the Woodward paradigm, Experiment 2 presented infants with object-directed reaches in three different locations: the left, middle, and right sides of the floor on which the actor was seated. During habituation, the two objects occupied two of the three locations and switched places between those locations in alternating habituation trials. Regardless of location, the actor always reached for the same object in habituation. In the test trials, the original object that the person had reached for was now in the third location, where no object had appeared during habituation. The other object, on which the person had never acted, was now in one of the two locations to which he had alternately reached on the previous habituation trials.

Otherwise, the displays from Experiment 2 closely mirrored those from Experiment 1. All habituation trials began with a person seated in front of two objects: a brown teddy bear and a blue origami ball. One object was located in the middle, directly in front of the person, whereas the other object was located to the left or right. The person looked to one of the objects, reached for it, and touched it. Upon touching the object, both objects changed color (they turned pink) and a bell sound played for 2.1 seconds, as though the person's actions had caused these changes in the object that he touched. Then, the person retracted his hand, and the two objects returned to their original colors as the sound stopped.

In alternating habituation trials, the two objects switched locations, and the person always reached for the same object, regardless of its location. 
In the single familiarization trial, the bear and the ball appeared, each on one side of the person. Importantly, the object that the person had reached for was on the side that he had not reached to before, and the other object was on the side that he had reached to on half the habituation trials.

The 6 test trials began with the person seated in front of the two objects, each now in their new locations. In alternating trials, the person either reached for the same object that he had reached for, presented at the new location, or for the other object, presented at a familiar location.

Procedure Data collection occurred during the COVID-19 pandemic and took place over Zoom video calls. Infants sat in their parents' laps, and viewed displays either on laptop screens $(n=22)$ or desktop screens $(n=2)$. Parents were instructed to sit quietly, not influence their infants, and look away from the displays in test trials. We used different software (jHab, Casstevens, 2007) to code infants' looking and presented the displays through Zoom rather than PyHab (used in Experiment 1 for in-lab testing), to minimize glitches and delays.

Habituation trials continued until infants met our habituation criterion: either the sum of the looking time on three consecutive trials after the first three were less than half the sum of the looking time on the first three, or 14 trials. To align infants' experiences better with the experiences of the 3-month-old infants tested in previous experiments using the Woodward paradigm (especially Sommerville et al., 2005), we increased both the maximum number of habituation trials (from 12 to 14) and the maximum duration of all infant controlled trials (from 45s to 120s). All other aspects of the procedure were identical to Experiment 1.

Looking time was assessed, as in habituation trials, by an online coder who was blind to the conditions and events. A second blind coder recoded $95 \%$ of test trials offline using Datavyu (2014) software. (One infant's looking could not be recoded because of an error in recording the session.) The intraclass correlation coefficient between the two observers' data was 0.97 (95\% CI [0.96,0.98]).

Counterbalancing As in Experiment 1, we counterbalanced the identity of the original object that the person reached for (ball or bear), the starting location of the original object (left or right), and the order of test trials (first reach to the original object vs. first reach to the new object). In the first habituation trial, the original goal object was located on either the left or the right; across habituation trials, it alternated between that location and the middle location.

\section{Results}

Preregistered Analyses As in Experiment 1, a mixed-effects model with the dependent variable of log-transformed looking time, the fixed effect of trial type (old or new object), and the random intercept of trial pair, with a by-participant random slope, served to analyze infants' looking times on the test trials. Infants looked longer, during test, at reaches to a different object $\left(\right.$ mean $\left._{\text {diff object }}=13.21 \mathrm{~s}\right)$ than at reaches to a different location $\left(\right.$ mean $\left._{\text {diff location }}=9.52 \mathrm{~s}\right)$ trials $(\beta=-0.38$, $95 \%$ CI of $\beta[-0.63,-0.14], b=-0.40, t(76)=-3.07, p=.002)$ (see Fig. 2).

We next checked for influential participants in this model using Cook's Distance, a method for outlier detection. This method identified two influential participants in our mixedeffects model. Excluding these participants from the analysis, we found that infants still looked longer at reaches to a different object than at reaches to a different location $(\beta=$ $0.30,95 \%$ CI of $\beta[-0.55,-0.04], b=-0.28, t(68)=-2.29, p=$ $.026)$.

Exploratory Analysis In an additional analysis, we tested whether the pattern of findings differed between Experiments 1 and 2, using a mixed effect model with the dependent variable of log-transformed looking time and the fixed effects of trial type, experiment, and the interaction between them. With the random intercept of participant ID, and fixed effects centered, the analysis yielded a significant effect of experiment $(\beta=-0.91,95 \%$ CI of $\beta[-1.26,-0.57], b=-0.92$, $t(42)=-5.21, p<.001)$, such that infants looked longer at test in Experiment $1\left(\operatorname{mean}_{\mathrm{E} 1}=21.42 \mathrm{~s}\right)$ than in Experiment 2 $\left(\right.$ mean $\left._{\mathrm{E} 2}=11.37 \mathrm{~s}\right)$. This could be due to differences in testing conditions (i.e. a large projector screen in a quiet, dark room in Experiment 1, vs. a much smaller screen in a far less controlled home environment in Experiment 2), the use of more and longer habituation trials in Experiment 2, or because infants in Experiment 1 did not know if the person's goal was defined over an object's intrinsic properties or location, and thus found both test events to be equally informative.

There was further a significant effect of trial type $(\beta=0.21$, $95 \%$ CI of $\beta[0.05,0.36], b=0.20, t(140)=2.65, p=.008)$, pooling data across both studies, such that infants looked longer at the test events in which the actor reached to the new object. However, this effect was qualified by an interaction $(\beta=0.38,95 \%$ CI of $\beta[0.07,0.69], b=0.38, t(140)=2.43$, $p=.016)$. Posthoc pairwise tests, correcting for multiple comparisons using Holm's method, revealed that the difference in infants' looking during "different object" and "different location" test trials was significant in Experiment $2(\beta=-0.39, b=-0.40, t(137)=-3.69, p<.001)$, but not in Experiment $1(\beta=-0.01, b=-0.01, t(139)=-0.14, p=.883)$.

\section{Discussion}

In Experiment 2, after seeing a person reach for the same object when it appeared in 2 different locations, 3-month-old infants looked longer during test trials when the person reached for a different object than when the person reached for the original object, even though it was now in a third, novel location. These findings suggest that when infants see other people reach for objects under conditions where an object's intrinsic properties are disambiguated from its location, they can appreciate that reaching is a goal-directed action, and that this goal can be a particular object. 


\section{General Discussion}

Before infants learn to reach for objects, what do they know about other people's reaching actions? Past research has shown that 3-month-old infants struggle to appreciate the goals behind other people's reaches. Here, we test whether infants truly fail to represent reaching as goal-directed more broadly, or whether they: (1) do not yet see reaching as an intentional causal action, or (2) understand that people reach with goals in mind, but do not yet know what these goals are. We found that when infants are given clear evidence that a person's reach has causal consequences (because the object changes its state on contact with the person's hand) and that the person reaches for the same object irrespective of its location (because the direction of his reach changes as the object changes location), they expect the person to continue reaching for that object, even when it appears for the first time on the opposite side of the array.

These findings build on evidence that 3-month-old infants, in the absence of first-person experience with reaching, can represent reaching as an intentional, costly, goal-directed action (Liu et al., 2019; Choi et al., 2018). Contrary to some views (e.g., Gallese \& Goldman, 1998; Meltzoff et al., 2009; Woodward, 2009), first-person experience reaching is not necessary for infants to see others' reaching as objectdirected. Three-month-old infants are not capable of reaching for objects themselves, but when given both causal information and information that disambiguated an object's location from its intrinsic properties, they can adaptively infer the goals of other people's reaching actions.

Together, these findings invite a reinterpretation of past findings that prereaching infants do not look differently following reaches to objects that a person has or has not reached for before (Gerson \& Woodward, 2014; Sommerville et al., 2005). Past work had concluded from these null findings that prereaching infants "fail to show sensitivity to others' reaching actions as goal-directed" (e.g., Woodward, 2013; see also, Filippi \& Woodward, 2015; Woodward, 2009). By contrast, the present findings suggest that prereaching infants are sensitive to the object-directed goals of others' actions, but that they are open-minded about whether an object's intrinsic properties or its location matter more to a person. When these two types of object properties are placed in conflict, 3-month-old infants require more information to support their predictions of other people's future actions.

In disambiguating an object's intrinsic properties from its location, we found evidence that 3-month-old infants represent object-directed goals, but two key questions remain. First, can infants attribute location goals to agents? If prereaching infants in past work did not demonstrate sensitivity to object goals because of conflicting information about which object properties are relevant to a person's goal, then infants may learn to attribute a location goal to an agent who reaches for whatever object is in a specific location, regardless of what object is there. We are currently testing this possibility in ongoing research.
Second, what is the role of causal information in 3-monthold infants' goal attribution? Although our first experiment suggests that causal information alone was not sufficient for 3-month-olds to attribute object goals, it is unknown if infants would learn to attribute object goals to an agent who reaches for one object in two locations, using the method of Experiment 2, without changing the state of the object.

In sum, our findings provide evidence that prereaching infants see others' reaches as reflecting their goals, but that they do not yet know whether an object's intrinsic properties or location are more relevant to the goals of others' reaching actions. Given that people's actions sometimes depend on objects' locations, especially for foraging human groups who learn to identify food sources by the locations of the plants that produce them, infants' open-mindedness to different goals may be an adaptive strategy. In industrialized societies, most of the objects that infants' encounter will have the same affordances wherever they are located. By observing people who act on these objects in diverse locations, infants may learn to weight intrinsic properties over locations in predicting others' object-directed actions.

What do 3-month-old infants see when watching other people act? On one view, infants see bodies moving through space, and are ignorant that intentions and goals guide other people's actions. Given the current findings, we submit that infants are not ignorant, but instead open-minded. Infants understand broadly that agents have goals, can produce costly actions, and act so as to cause these goals to become real. Given this understanding, infants still have to learn what people want, which actions help them get what they want, and how. These early-emerging over-hypotheses about how agents work could serve to help infants to learn rapidly about other people's actions and minds.

\section{Acknowledgements}

We thank the families who participated; Bill Pepe for research assistance; Manasa Ganesh Kumar and Maria Castro Roldán for help with data coding; Nensi Gjata and Michael Gajda for acting in stimuli; and Hyowon Gweon and the Stanford Social Learning Lab for sharing protocols to facilitate online testing. This material is based on work supported by the Center for Brains, Minds, and Machines, funded by National Science Foundation STC Award CCF1231216; by Siegel Foundation Award S4881; by an NSF Graduate Research Fellowship under grant DGE-1144152 (SL); by an NIH NRSA Postdoctoral Fellowship under grant F32HD103363 (SL); and by a Social Sciences and Humanities Research Council Doctoral Fellowship under award 752-2020-0474 (BW).

\section{References}

Baker, C. L., Saxe, R., \& Tenenbaum, J. B. (2009). Action understanding as inverse planning. Cognition, 113(3), 329-349.

Biro, S., \& Leslie, A. M. (2007). Infants' perception of goaldirected actions: Development through cue-based bootstrapping. Developmental Science, 10(3), 379-398. 
Casstevens, R. M. (2007). jHab: Java habituation software (version 1.0. 2) [computer software]. Chevy Chase, MD.

Choi, Y. J., Mou, Y., \& Luo, Y. (2018). How do 3-month-old infants attribute preferences to a human agent? Journal of Experimental Child Psychology, 172, 96-106.

Datavyu Team. (2014). Datavyu: A video coding tool. Retrieved from http://datavyu.org

Daum, M., Attig, M., Gunawan, R., Prinz, W., \& Gredebäck, G. (2012). Actions seen through babies' eyes: A dissociation between looking time and predictive gaze. Frontiers in Psychology, 3, 370.

Feiman, R., Carey, S., \& Cushman, F. (2015). Infants' representations of others' goals: Representing approach over avoidance. Cognition, 136, 204-214.

Gallese, V., \& Goldman, A. (1998). Mirror neurons and the simulation theory of mind-reading. Trends in Cognitive Sciences, 2(12), 493-501.

Gerson, S. A., \& Woodward, A. L. (2014a). Learning from their own actions: The unique effect of producing actions on infants' action understanding. Child Development, 85(1), 264-277.

Gerson, S. A., \& Woodward, A. L. (2014b). The joint role of trained, untrained, and observed actions at the origins of goal recognition. Infant Behavior and Development, 37(1), 94-104.

Jara-Ettinger, J., Schulz, L. E., \& Tenenbaum, J. B. (2020). The naive utility calculus as a unified, quantitative framework for action understanding. Cognitive Psychology, 123, 101334.

Hernik, M., \& Southgate, V. (2012). Nine-months-old infants do not need to know what the agent prefers in order to reason about its goals: On the role of preference and persistence in infants' goal-attribution. Developmental Science, 15(5), 714-722.

Kominsky, J. F. (2019). PyHab: Open-source real time infant gaze coding and stimulus presentation software. Infant Behavior and Development, 54, 114-119.

Liu, S., Brooks, N. B., \& Spelke, E. S. (2019). Origins of the concepts cause, cost, and goal in prereaching infants. Proceedings of the National Academy of Sciences, 116(36), 17747-17752.

Luo, Y., \& Johnson, S. C. (2009). Recognizing the role of perception in action at 6 months. Developmental Science, 12(1), 142-149.

Sommerville, J. A., Woodward, A. L., \& Needham, A. (2005). Action experience alters 3-month-old infants' perception of others' actions. Cognition, 96(1), B1-B11.

Tomasello, M., Carpenter, M., Call, J., Behne, T., \& Moll, H. (2005). Understanding and sharing intentions: The origins of cultural cognition. Behavioral and Brain Sciences, 28(5), 675-691.

Von Hofsten, C. (2004). An action perspective on motor development. Trends in Cognitive Sciences, 8(6), 266-272.

Woodward, A. L. (1998). Infants selectively encode the goal object of an actor's reach. Cognition, 69(1), 1-34.
Woodward, A. L. (2009). Infants' grasp of others' intentions. Current Directions in Psychological Science, 18(1), 5357. https://doi.org/10.1111/j.1467-8721.2009.01605.x

Woodward, A. L. (2013). Infant foundations of intentional understanding. In M. R. Banaji \& S. A. Gelman (Eds.), Navigating the Social World (pp. 75-80). Oxford University Press. 